\title{
Giant Lipoma Extending Between the Heads of Biceps Brachii Muscle and the Deltoid Muscle: Case Report
}

\author{
Svetoslav Slavchev ${ }^{\mathrm{a}}$, Georgi P. Georgiev ${ }^{\mathrm{a}, \mathrm{d}}$, Marin Penkov ${ }^{\mathrm{b}}$, Boycho Landzhov ${ }^{\mathrm{c}}$
}

\begin{abstract}
Lipomas are common benign neoplasms consisting of mature fatty tissue. They are usually of roundish or ovoid shape and are situated in a single anatomical region. They most frequently occur on the back and in the extremities. We present a rare case of a giant hourglass-shaped lipoma displacing the axillary neuro-vascular bundle, extending between the heads of the biceps brachii muscle, and distending the deltoid muscle. The lipoma was excised through a deltoidopectoral approach with uneventful healing. The radiological and MRI findings and surgical challenges are briefly discussed.
\end{abstract}

Keywords: Giant lipoma; Shoulder joint; Radiological appearance; Surgery

\section{Introduction}

Lipomas are the most frequent benign mesenchymal neoplasms with an estimated incidence of about 16\% [1-3]. They consist of mature fatty tissue and are believed to arise from primordial adipocytes rather than from adult fat cells [1]. Lipomas most frequently occur in the back and in the extremities as well-circumscribed, encapsulated roundish or ovoid subcutaneous movable masses $[1,2,4]$. The patients' complaints are typically of slow growing painless tumor causing aesthetic defects and only rarely of compression symptoms of neural or vascular origin $[1,5]$. Lipomas typically reach a

\footnotetext{
Manuscript accepted for publication June 7, 2012

aUniversity Hospital of Orthopaedics "Prof. B. Boychev", Sofia, Bulgaria

bUniversity Hospital "Saint Ivan Rilski”, Sofia, Bulgaria

${ }^{\mathrm{c}}$ Department of Anatomy, Histology and Embryology, Medical

University-Sofia, Bulgaria

${ }^{\mathrm{d}}$ Corresponding author: Georgi P. Georgiev, University Hospital of Orthopaedics, Medical University-Sofia, 56 Nicola Petkov Blvd,

BG-1614 Sofia, Bulgaria. Email: georgievgp@yahoo.com
}

doi: http://dx.doi.org/10.4021/jcs $84 \mathrm{w}$ diameter of several centimeters and are localized in a single anatomical region $[3,4]$.

Herein, we present an extremely rare case of a giant hourglass-shaped lipoma in two anatomical regions, the axilla and the deltoid region, that displaces the axillary neurovascular bundle, extends between the heads of the biceps brachii muscle, and distends the deltoid muscle. The lipoma was excised through a deltoidopectoral approach with uneventful healing.

\section{Case Report}

A 73-years old woman presented at our institution with a painless mass in her left shoulder region that she had noticed about two months earlier and that caused no other symptoms. On clinical examination the left deltoid region was visibly expanded in its anterior portion and a palpable nontender mass was present extending into the axilla. Pulsations of the axillary artery could be followed over the axillary mass. Plain radiographs revealed a well-delineated ovoid radionegative tumor situated medial and lateral to the humerus that was diagnosed as a lipoma (Fig. 1a). On MRI the tumor presented as a homogenous hourglass-shaped mass, displacing the axillary neuro-vascular bundle distally and medially towards the chest wall, extending between the long head of the biceps brachii muscle that runs deep to the isthmus of the lipoma and the coracobrachialis muscle and the short head of the biceps brachii superficial to it, and distending the deltoid muscle in an anterior direction (Fig. 1b, c). The maximum diameter of the lipoma was measured to be $10.4 \mathrm{~cm}$.

Surgery was performed through a deltoidopectoral approach. After the deltoid muscle was retracted and the lateral lobe of the lipoma was mobilized, the conjoint tendon had to be detached from the coracoid process to allow for safe mobilization of the isthmus without forceful retraction and strain to the musculocutaneous nerve (Fig. 2a, b). The medial lobe of the lipoma was mobilized by blunt dissection from behind the pectorals major muscle and although its humeral insertion was an obstacle towards the axilla, it still allowed for removal of the lipoma without being desinserted. After the lipoma was excised, the conjoint tendon was reinserted 

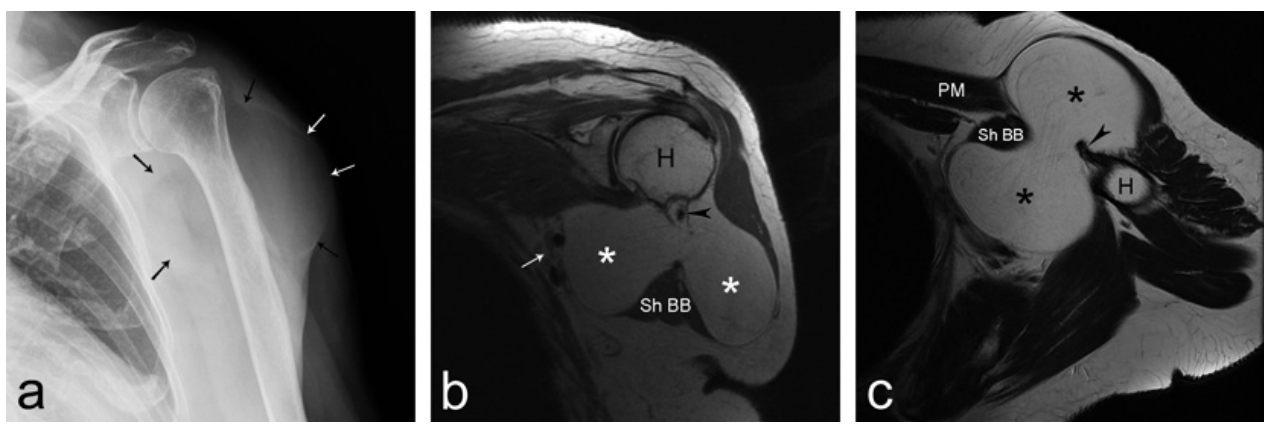

Figure 1. a: Plain radiograph showing radionegative well delineated mass (arrows); b and c: MRI scan presenting a homogenous bi-lobed non-infiltrative tumor (asterisks) displacing the neurovascular bundle medially (arrows). Muscles: tendon of the long head of biceps brachii (arrowheads); pectoralis major muscle (PM); short head of biceps brachii muscle (Sh BB); Bone: humerus $(\mathrm{H})$.

and the wound was closed over a suction drain in the usual manner. The wound healing was uneventful. The excised lipoma weighed 170 grams (Fig. 2c).

Histological examination of the specimen confirmed a benign lipoma. Postoperative course was uneventful and twelve months after surgery no clinical recurrence was detected.

\section{Discussion}

Lipomas are considered as "one of the most innocent of tumours" that usually grow slowly and do not infiltrate the neighbouring structures $[3,5]$. Most common the lipomas remain asymptomatic or presenting a cosmetic problem, and rarely could cause limitation of motion, lymphoedema or neurovascular compression, especially in cases of so called "giant lipomas" $[1-3,5]$. There is discordance in the literature about the size that defines giant lipomas. Some advocate $10 \mathrm{~cm}$ in any dimension as the margin above which a lipoma is considered to be giant as in our case [2,4], while others accept $5 \mathrm{~cm}$ in the upper extremity [1]. It seems intuitively clear that a "subgiant" lipoma might cause aesthetic or clinical complaints in a slender woman's hand or go unnoticed for a long time in a large man's arm. Therefore, we think it may be more appropriate to classify lipomas as giant when they approach or exceed the normal size of the anatomical region they are situated in.

The exact mechanism for the increased growth of such lipomas is still in debate [3,4]. Most authors propose microtrauma due to the great range of motion in the axilla that causes rupture of the fibrous septa and anchorage connections between the skin and deep fascia thus allowing the adipose tissue to proliferate [1-3]. Other theories, including the role of endocrine, dysmetabolic and genetic factors provoking the uncontrolled growth of giant cell lipomas, have been also proposed [5].

Preoperative imaging studies reveal the extent of the lesion and facilitate the operative planning [1]. Radiographs often presented the lipoma as a well-delineated radionegative tumor. On MRI and CT the lipoma appears as a non-invasive mass with homogenous fat signal intensity enveloped by a pseudocapsule [1]. Ultrasound investigations could also been used in detection of lipomas [1, 3, 4]. In cases of malignancy, MRI and CT could distinguish a lipoma from a welldifferentiated liposarcoma because of the increased levels of vascularity seen in septal structures within the malignant lesion [1].

The treatment of giant lipomas consists of complete surgical removal [1-4]. Lipomas are usually well-encapsulated, thus allowing relatively easy complete excision $[1,2]$. Intralesional removal through liposuction has also been reported $[3,4]$. However, it is associated with higher frequency of local recurrence and a higher risk of neural or vascular injury
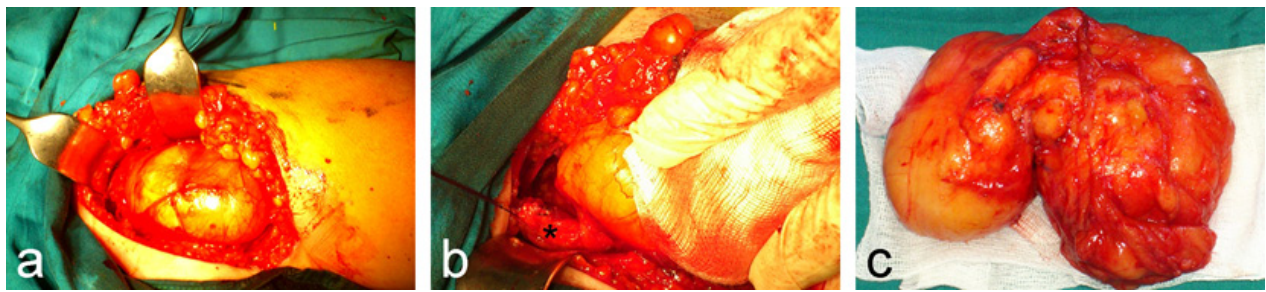

Figure 2. a: Intraoperative findings after deltoidopectoral approach; b: Conjoint tendon detached from coracoid process; c: Excised lipoma. 


\section{$[1,3]$}

\section{Conflict of Interest Statement}

No conflicts declared.

\section{References}

1. Allen B, Rader C, Babigian A. Giant lipomas of the up- per extremity. Can J Plast Surg. 2007;15(3):141-144.

2. Copcu E, Sivrioglu N, Culhaci N. Axillary giant lipoma. Plast Reconstr Surg. 2004;114(7):1982-1983.

3. Vandeweyer E, Scagnol I. Axillary giant lipoma: a case report. Acta Chir Belg. 2005;105(6):656-657.

4. Huang JC, Hsu KF, Hsu HM, Chan DC, Yu JC. Giant Axillary Lipoma. J Med Sci. 2009;29(4):213-215.

5. Sergeant G, Gheysens O, Seynaeve P, Van Cauwelaert J, Ceuppens H. Neurovascular compression by a subpectoral lipoma. A case report of a rare cause of thoracic outlet syndrome. Acta Chir Belg. 2003;103(5):528-531. 